\title{
AN EFFICIENT REDUCED-ORDER MODEL FOR THE NONLINEAR DYNAMICS OF CARBON NANOTUBES
}

\author{
Tiantian Xu \\ Department of Mechanical \\ Engineering \\ State University of New York \\ Binghamton, NY, USA \\ txu2@binghamton.edu
}

\author{
Mohammad I. Younis \\ Department of Mechanical \\ Engineering \\ State University of New York \\ Binghamton, NY, USA \\ myounis@binghamton.edu \\ Physical Sciences and \\ Engineering Division \\ King Abdullah University of \\ Science and Technology \\ (KAUST), Thuwal 23955-6900, \\ Saudi Arabia \\ mohammad.younis@kaust.edu.

\begin{abstract}
Because of the inherent nonlinearities involving the behavior of CNTs when excited by electrostatic forces, modeling and simulating their behavior is challenging. The complicated form of the electrostatic force describing the interaction of their cylindrical shape, forming upper electrodes, to lower electrodes poises serious computational challenges. This presents an obstacle against applying and using several nonlinear dynamics tools that typically used to analyze the behavior of complicated nonlinear systems, such as shooting, continuation, and integrity analysis techniques. This works presents an attempt to resolve this issue. We present an investigation of the nonlinear dynamics of carbon nanotubes when actuated by large electrostatic forces. We study expanding the complicated form of the electrostatic force into enough number of terms of the Taylor series. We plot and compare the expanded form of the electrostatic force to the exact form and found that at least twenty terms are needed to capture accurately the strong nonlinear form of the force over the full range of motion. Then, we utilize this form along with an
\end{abstract}

Euler-Bernoulli beam model to study the static and dynamic behavior of CNTs. The geometric nonlinearity and the nonlinear electrostatic force are considered. An efficient reduced-order model (ROM) based on the Galerkin method is developed and utilized to simulate the static and dynamic responses of the CNTs. We found that the use of the new expanded form of the electrostatic force enables avoiding the cumbersome evaluation of the spatial integrals involving the electrostatic force during the modal projection procedure in the Galerkin method, which needs to be done at every time step. Hence, the new method proves to be much more efficient computationally.

\section{INTRODUCTION}

Nanoelectromechanical systems (NEMS) are a growing area of research, which extends the microelectromechanical systems (MEMS) technology to the nanoscale [4,5]. Research on NEMS is developing very quickly. Currently NEMS are largely applied in a variety of different fields, e.g. communications, industrial, bioengineering, etc., where they cover many different functions, including sensing and 
actuation. Their mechanical behavior analysis is a difficult and challenging task [6, 7]. Carbon nanotubes (CNTs), as one of the most promising kind of NEMS application have recently been the subject of much exciting research due to their unique electrical and mechanical properties. More in depth research however is needed for the mechanical response of CNTs to be able to implement them as functional devices and to utilize them as resonant sensors, switches, and actuators. Particularly, extensive and accurate nonlinear dynamic analysis is needed to explain many of the reported experimental data and to reveal the full potential of CNTs when employed in device applications [1-3]. Beside their interesting mechanical properties, carbon nanotubes inherit their remarkable electrical properties from the unique electronic band structure of graphene. Electrically actuated CNTs were shown to have rich nonlinear behavior characterized by inherent nonlinear response even for small electric loads [8] as well as a wide range of tunability of their natural frequencies with the DC load [9].

Many investigations on the vibration of CNTs under several dynamic loadings [4-19] have been done by several groups all over the world. For example, Ouakad and Younis [1] investigated using a Galerkin procedure the nonlinear dynamic behavior of electrically actuated CNT resonators. They showed complex nonlinear dynamics phenomena, such as hysteresis, dynamic pullin, hardening and softening behaviors, and frequency bands with an inevitable escape from a potential well.

Most previous simulation studies model clamped-clamped CNTs to be perfectly straight. However, in practical fabrication process, fabricating perfectly straight CNTs with controlled geometry and orientation is hard. Actually, clamped-clamped CNTs are usually fabricated with some level of curvature (slack) [10-15]. Thus, it is important and instructive to model the mechanics of initially slacked CNTs as Gibson indicated in his study [10]. Experimental investigations by Sazonova et al. [11] and Sazonova [12] also show the importance of slack on estimating accurately the vibration behavior of CNTs.

It is well known that curvature of arches introduces quadratic and cubic nonlinearities. Furthermore, electrostatic forces are inherently nonlinear. The interaction of these nonlinearities (how they compete, strengthen, or cancel each other) still is not fully understood. Ouakad and Younis [2,3] investigated in depth the nonlinearities of single-walled CNTs and their effects on the dynamics while varying the slack level and the DC voltages.

Because of the inherent nonlinearities involving the behavior of CNTs when excited by electrostatic forces, modeling and simulating their behavior is challenging. The complicated form of the electrostatic force describing the interaction of their cylindrical shape, forming upper electrodes, to lower electrodes poises serious computational challenges. This presents an obstacle against applying and using several nonlinear dynamics tools that typically used to analyze the behavior of complicated nonlinear systems, such as shooting, continuation, and integrity analysis techniques. The objective of this paper is to investigate an efficient model to simulate the nonlinear dynamic behavior of CNTs with different slack level under a wide range of voltage loads.

\section{PROBLEM FORMULATION}

In this section, we formulate the problem governing the static and dynamic behavior of an electrically actuated slacked single-walled carbon nanotube, Fig. 1 . The nanotube is actuated by an electrode underneath it with a gap width $d$. It is modeled as a cylindrical Euler-Bernoulli beam of radius $\tilde{R}$, shell thickness $h$, and length $L$. It has a cross-sectional area $A$ and an area moment of inertia $I$. The nanotube is assumed to have a Young modulus $E$ and a mass density $\rho$. The CNT is considered here to be initially curved in the direction of the lower electrode with an initial shape

$$
\hat{w}_{0}(\hat{x})=b_{0} \sin ^{2}\left(\frac{\pi \hat{x}}{L}\right)
$$

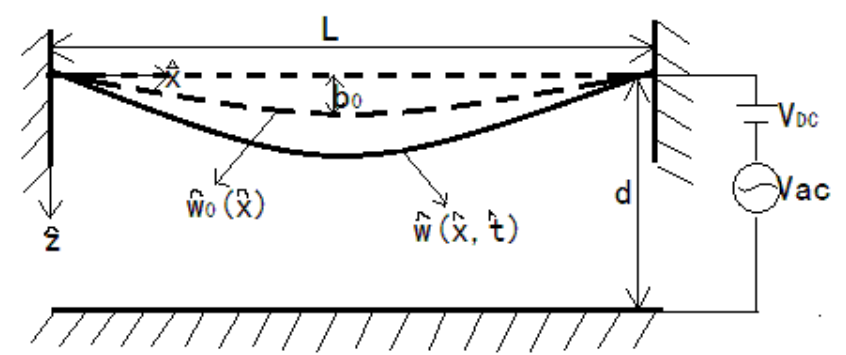

Fig. 1 Schematic of initially curved carbon nanotube

The equation describing the deflection $\hat{w}(\hat{x}, \hat{t})$ of the clamped-clamped CNT can be written as $[2,3]$

$$
\begin{aligned}
& E I \frac{\partial^{4} \hat{w}}{\partial \hat{x}^{4}}+\rho A \frac{\partial^{2} \hat{w}}{\partial \hat{t}^{2}}+\tilde{c} \frac{\partial \hat{w}}{\partial \hat{t}}= \\
& \frac{E A}{2 L}\left[\int_{0}^{L}\left\{\left(\frac{\partial \hat{w}}{\partial \hat{x}}\right)^{2}-2\left(\frac{\partial \hat{w}}{\partial \hat{x}}\right)\right\} d x\right] \times\left[\frac{\partial^{2} \hat{w}}{\partial \hat{x}^{2}}-\frac{d^{2} \hat{w}_{0}}{d \hat{x}^{2}}\right]+\hat{F}_{e}
\end{aligned}
$$

where the electrostatic force $\hat{F}_{e}$ is expressed as [16]

$$
\frac{\hat{F}_{e}=}{\sqrt{\left(d-\hat{w}-\hat{w}_{0}\right)\left(d-\hat{w}-\hat{w}_{0}+2 \tilde{R}\right)\left(\cosh ^{-1}\left(1+\frac{d-\hat{w}-\hat{w}_{0}}{\tilde{R}}\right)\right)^{2}}}
$$

In (1), $\hat{t}$ is time, $\tilde{c}$ is the viscous damping coefficient, and $\varepsilon_{0}$ is the air permittivity.

The boundary conditions are:

$$
\hat{w}(0, \hat{t})=0, \frac{\partial \hat{w}}{\partial \hat{x}}(0, \hat{t})=0 \quad \hat{w}(L, \hat{t})=0, \frac{\partial \hat{w}}{\partial \hat{x}}(L, \hat{t})=0 .
$$


Before proceeding further, it should be mentioned that the continuum mechanics beam theory of (1) is valid as long as the following conditions are satisfied $[2,18,19]$ :

$a_{c} / L<<1$
$d_{N T} / L<<1$

Where $d_{N T}$ is the CNT diameter and $a_{c}$ is the width of the hexagonal carbon rings, which is estimated to be near $0.24 \mathrm{~nm}$. In addition, to comply with the shallow arch theory, the initial curvature of the arch (the slack) needs to remain small, i.e., $b_{0} / L<0.1$. For the considered case studies of this paper, these conditions are satisfied.

For convenience, we introduce the following nondimensional variables:

$$
w=\frac{\hat{w}}{d} \quad x=\frac{\hat{x}}{L}, \quad t=\frac{\hat{t}}{T}
$$

where $T$ is a time constant defined by $T=\sqrt{\left(\rho A L^{4}\right) /(E I)}$. By substituting (4) into (1)-(3), the nondimensional equations of motion and associated boundary conditions of the considered clamped-clamped CNT are written as:

$$
\begin{aligned}
& \frac{\partial^{4} w}{\partial x^{4}}+\frac{\partial^{2} w}{\partial t^{2}}+c \frac{\partial w}{\partial t}= \\
& \alpha_{2} F e+\alpha_{1}\left[\int_{0}^{1}\left\{\left(\frac{\partial w}{\partial x}\right)^{2}-2\left(\frac{\partial w}{\partial x} \frac{d w_{0}}{d x}\right)\right\} d x\right] \times\left[\frac{\partial^{2} w}{\partial x^{2}}-\frac{d^{2} w_{0}}{d x^{2}}\right] \\
& w(0, t)=0, \frac{\partial w}{\partial x}(0, t)=0, w(1, t)=0, \frac{\partial w}{\partial x}(1, t)=0
\end{aligned}
$$

Where

$$
F_{e}=\frac{\pi \varepsilon_{0}\left(V_{D C}+V_{A C} \cos (\Omega t)\right)^{2}}{\sqrt{\left(d-w-w_{0}\right)\left(d-w-w_{0}+2 R\right)\left(\cosh ^{-1}\left(1+\frac{d-w-w_{0}}{R}\right)\right)^{2}}}
$$

$$
w_{0}(x)=\frac{b_{0}}{d} \sin ^{2}\left(\frac{\pi x}{L}\right)
$$$$
\alpha_{1}=\frac{A d^{2}}{2 I} \quad \alpha_{2}=\frac{\pi \varepsilon_{0} L^{2}}{E I d^{2}} \quad c=\frac{\tilde{c} L^{4}}{E I} \text {, }
$$

$\Omega=\frac{\tilde{\Omega}}{\sqrt{(E I) /\left(\rho A L^{4}\right)}} \quad R=\frac{\tilde{R}}{d}$

\section{TAYLOR EXPANSION}

To reduce computational difficulty, we attempt to expand the complicated form of the electrostatic force into enough number of terms of the Taylor series.

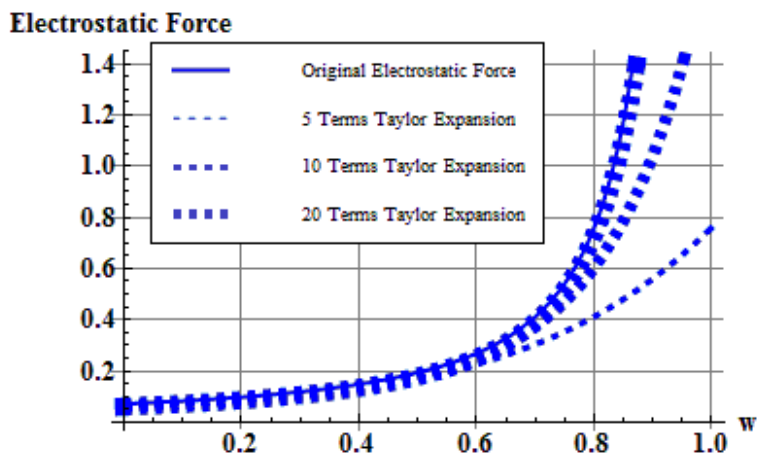

Fig.2 Taylor series expansion of the nondimentional electrostatic force.

From Fig.2 we can see that by comparing the expanded form of the electrostatic force to the exact form, at least twenty terms are needed to capture accurately the strong nonlinear form of the force over the full range of motion. Thus, we express the electrostatic force as

$F_{e} T_{20}=a_{0}+a_{1} w+a_{2} w^{2}+a_{3} w^{3}+\cdots$

$+a_{i} w^{i}+\cdots+a_{20} w^{20}$

Where $a_{0}, a_{1} \ldots \ldots a_{20}$ are Taylor expansion coefficients which are constants.

Then we substitute $F_{e} T_{20}$ for $F_{e}$ in (5) and get:

$\frac{\partial^{4} w}{\partial x^{4}}+\frac{\partial^{2} w}{\partial t^{2}}+c \frac{\partial w}{\partial t}=$

$\alpha_{2} F_{e} T_{20}+\alpha_{1}\left[\int_{0}^{1}\left\{\left(\frac{\partial w}{\partial x}\right)^{2}-2\left(\frac{\partial w}{\partial x} \frac{d w_{0}}{d x}\right)\right\} d x\right] \times\left[\frac{\partial^{2} w}{\partial x^{2}}-\frac{d^{2} w_{0}}{d x^{2}}\right]$

\section{THE REDUCED-ORDER MODEL}

To solve the obtained nondimensional equation of motion with its associated boundary conditions of the slacked CNT, (5)-(7b) are discretized using the Galerkin procedure to yield a Reduced-Order Model (ROM) $[1,20]$. Hence, the response of the CNT is approximated as

$$
w(x, t)=\sum_{i=1}^{n} u_{i}(t) \phi_{i}(x)
$$

Where $\phi_{i}(x)$ are the normalized linear undamped mode shapes of a straight beam and $u_{i}(t)$ are the nondimensional modal coordinates.

To obtain the ROM, we substitute (11) into (5)-(7b), multiply by $\phi_{i}(x)$, use the orthogonality conditions of the mode shapes, and then integrate the outcome from 0 to 1 . The results are differential equations in terms of the modal 
coordinates $u_{i}(t)$. To simulate the dynamic behavior, the obtained ROM can be integrated in time using Runge-Kutta technique.

Without substituting the electrostatic force with its Taylor expansion, the mode shapes $\phi_{i}(x)$ will remain embedded inside the denominator of the electrostatic force term of (7a) in the ROM. To deal with the complicated integral terms due to that electrostatic force, we have to evaluate the spatial integrals containing the mode shapes $\phi_{i}(x)$ numerically simultaneously while integrating the differential equations of the modal coordinates $u_{i}(t)$.

By using the Taylor series to replace the original electrostatic force, we could avoid the cumbersome evaluation of the spatial integrals involving the electrostatic force during the modal projection procedure in the Galerkin method, which needs to be done at every time step [1].

\section{LONG TIME INTEGRATION RESULTS}

As a case study, a non-hollow CNT of $L=1000 \mathrm{~nm}$, $\tilde{R}=5 \mathrm{~nm}, d=200 \mathrm{~nm}$, Young modulus $E=1 \mathrm{TPa}$, and mass density $\rho=1.3 \mathrm{~g} / \mathrm{cm}^{3}$ is considered.

Fig. 3 shows the maximum frequency response of the CNT. Numerical response with the same parameter calculated without the Taylor expansion from previous study of [3] are plotted in the same figure with numerical results using the simplified governing equation with the electrostatic force term substituted as its Taylor series. It has been mentioned in [3] that to get this result is quite computational challenging and time consuming due to the complex electrostatic force term. Using our method, results were obtained fast.

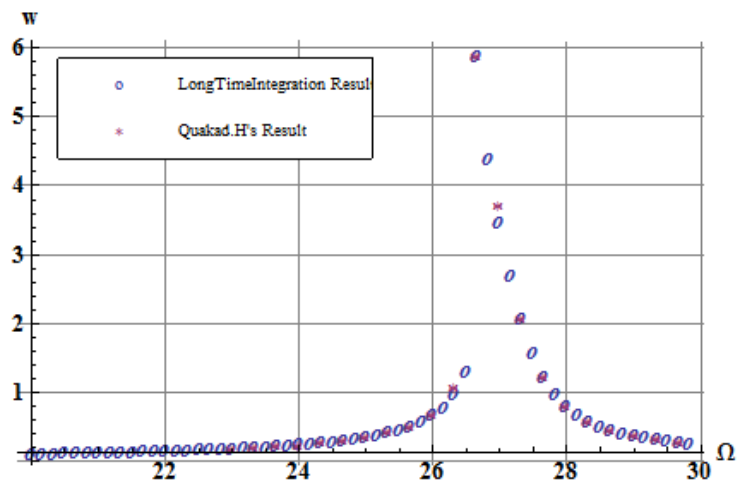

(a)

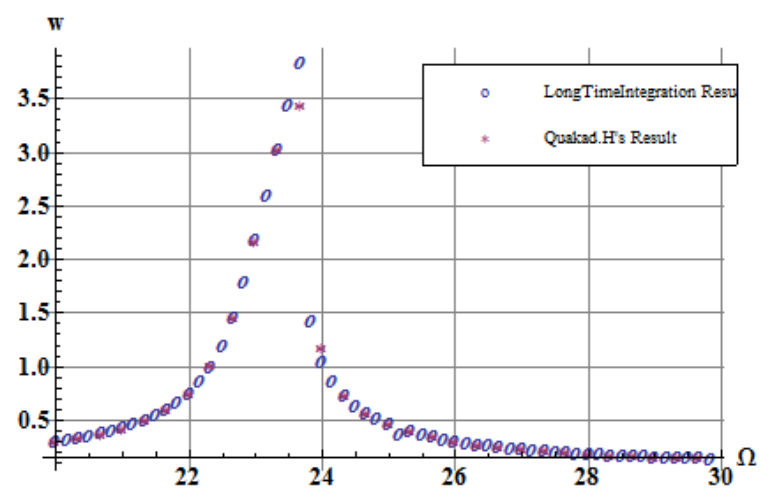

(b)

Fig. 3 Frequency-response curves of (a) $2 \mathrm{~nm}$ and (b) $5 \mathrm{~nm}$ slacked CNT showing hardening and softening behaviors respectively.

Results are shown for $V_{\mathrm{DC}}=V_{\mathrm{AC}}=1 \mathrm{~V}$ and a quality factor of 100

\section{SHOOTING METHOD}

The long-time integration technique may not predict accurately the dynamical behavior, especially near bifurcation points. Toward this, we use the shooting method [21]. The shooting method is a powerful technique for capturing periodic motion and analyzing their stability for nonlinear systems. Hence, it is capable of predicting both stable and unstable solutions. In this section, we utilize the shooting method in conjunction with the Floquet theory to obtain both stable and unstable solution of the frequency response of the CNT excited by electrostatic force.

We consider the same case study of Section 5. First we study the advantage of shooting method on revealing the nonlinear behavior of CNT under a certain electrostatic force. Fig. 4 shows that the shooting method result and longtime integration method has a good match. Moreover, shooting method could give us not only unstable solution which 
longtime integration method could not but it could show more comprehensive view of the entire range of the stable solution.

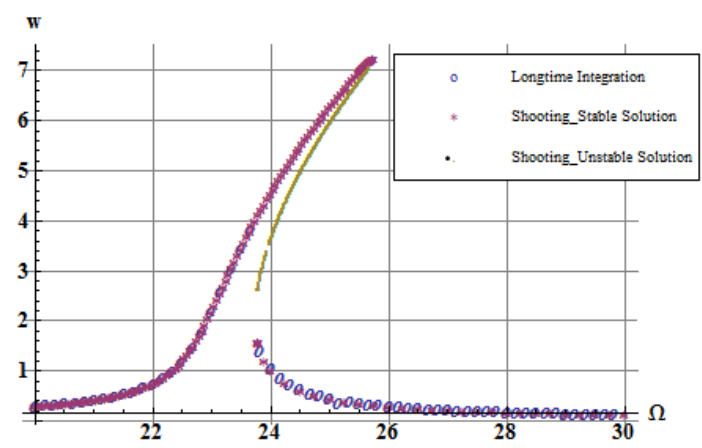

Fig.4. Frequency response curve of $2 \mathrm{~nm}$ slacked CNT. Results are shown for $V_{D C}=V_{A C}=1 V$ and a quality factor of 100 .

Next, we use this shooting technique to obtain more results. Fig.5 shows the response of a $5 \mathrm{~nm}$ slacked CNT under $V_{D C}=V_{A C}=1 V$ electrostatic force. The figure indicates softening behavior. Fig.6 shows the response of the $5 \mathrm{~nm}$ slacked CNT under large load, $V_{D C}=V_{A C}=5 \mathrm{~V}$. We could see a complicated nonlinear behavior in Fig.5 which is the CNT shows a general softening behavior and a local hardening behavior at around $\Omega=26.5 \mathrm{~Hz}$. More impressive, the figure reveals the existence of another stable solution of larger amplitude. Similar results were revealed recently using continuation techniques [22].

In the present research in this area, nonlinear behavior of slacked CNTs under large electrostatic force is missing. We can easily do simulation work with this efficient modal. In Fig.6, we could see an interesting phenomenon that there are two stable branch of solution at lower frequency.

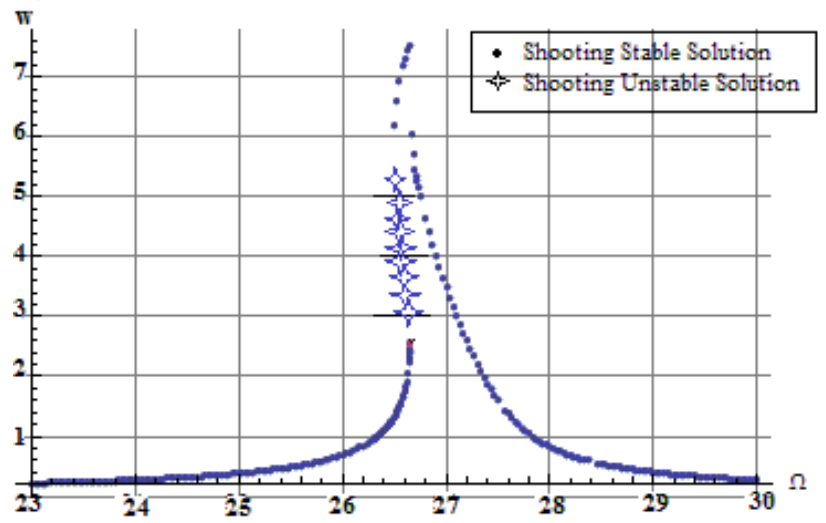

Fig. 5 Frequency-response curves of $5 \mathrm{~nm}$ slacked CNT.

Results are shown for $\mathrm{WDC}=V_{\mathrm{AC}}=1 \mathrm{~V}$ and a quality factor of 100 .

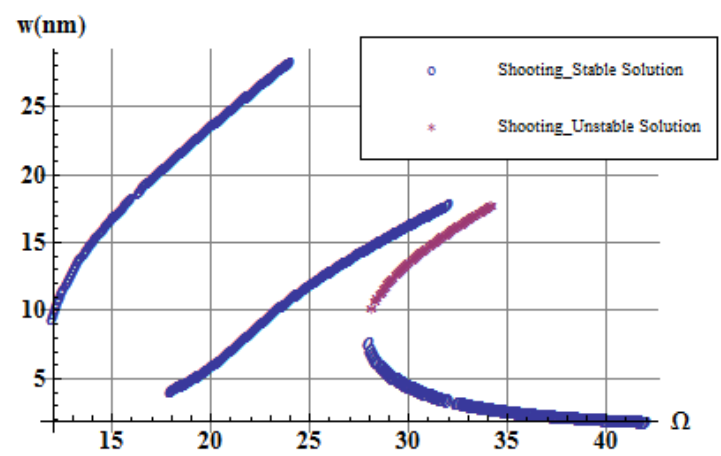

Fig. 6 Frequency-response curves of 5 nm slacked CNT.

Results are shown for $V_{\mathrm{DC}}=V_{\mathrm{AC}}=5 \mathrm{~V}$ and a quality factor of 100 .

\section{CONCLUSIONS}

In this paper, an investigation into the dynamic behavior of an electrically actuated slacked single-walled CNT when actuated by a DC force and an AC harmonic load was presented. Taylor expansion has been used to substitute the complicated electrostatic force term. It has been proven that this substitution could make the numerical simulation process much more efficient and would not affect the accuracy of the result.

Moreover, this efficient modal gives us a chance to simulate more result under wider range of electrostatic force load. We found that there are some unique and interesting nonlinear behaviors of CNTs when actuated by larger electrostatic force, which have never been studied before. Understanding of these phenomena needs further analysis and in-depth investigations.

\section{REFERENCES}

1. Ouakad, H. and Younis, M. I, "Nonlinear Dynamics of Electrically Actuated Carbon Nanotube Resonators", J. of Comp. and Non. Dyn., Vol. 5, \# 011009, 13 pages, 2010.

2. Ouakad, H. and Younis, M. I, "Natural frequencies and mode shapes of initially curved carbon nanotube resonators under electric excitation", J. of Sound and Vibrations, doi:10.1016/j.jsv.2010.12.029, Vol. 330, 3182-3195, 2011.

3. Ouakad, H. and Younis, M. I, "Dynamic Response of Slacked Carbon Nanotube Resonators," Non. Dyn., 67:1419-1436, DOI 10.1007/s11071-011-0078-3, 2012.

4. Pelesko, J. A. and Bernstein, D.H., 2003, Modeling MEMS and NEMS, Chapman \& Hall/CRC Press.

5. Gibson, R. F., Ayorinde, E. O., and Wen, Y. F., 2007, "Vibrations of Carbon Nanotubes and Their Composites: A Review," Compos. Sci. Technol., 67, pp. 1-28.

6. Rhoads, J. F., Shaw S. W., Turner, K. L., "Nonlinear 
Dynamics and Its Applications in Micro- and Nanoresonators," J. Dynamic Systems, Measurement, and Control, 2010, 132, 034001-1

7. Lifshitz, R., and Cross, M. C., 2008, "Nonlinear Dynamics of Nanomechanical and Micromechanical Resonators," Review of Nonlinear Dynamics and Complexity, 1, H. G. Schuster, ed., pp. 1-52.

8. Postma, H., Kozinsky, I., Husain, A., Roukes,M.: Dynamic range of nanotube- and nanowire-based electromechanical systems. Appl. Phys. Lett. 86, 223105 (2005)

9. Dequesnes, M., Tang, S., Aluru, N.R.: Static and dynamic analysis of carbon nanotube-based switches. J. Eng. Mater. Technol. 126, 230-237 (2004)

10. Gibson, R.F., Ayorinde, E.O., Wen, Y.F.:Vibrations of carbonnanotubes and their composites: a review. J. Comput Sci. Technol. 67, 1-28 (2007)

11. Sazonova, V., Yaish, Y., Üstünel, H., Roundy, D., Arias. T.A.,McEuen, P.L.: A tunable carbon nanotubes electromechanical oscillator. Nature 431, 284-287 (2004)

12. Sazonova, V.A.: A tunable carbon nanotube resonator. Ph.D. Thesis, Department of Physics, Cornell University.(2006)

13. Üstünel, H., Roundy, D., Arias, T.A.: Modeling a suspended nanotube oscillator. Nano Lett. 5, 523-526 (2005)

14. Garcia-Sanchez, D., San Paulo, A., Esplandiu, M.J., PerezMurano, F., Forrò, L., Aguasca, A., Bachtold, A.: Mechanical detection of carbon nanotube resonator vibrations. Phys. Rev. Lett. 99, 085501 (2007)

15. Mayoof, F.N., Hawwa, M.A.: Chaotic behavior of a curved carbon nanotube under harmonic excitation. Chaos Solitons Fractals 42, 1860-1867 (2009)

16. Ke, C.H., Espinosa, H.D., Pugno, N.: Numerical analysis of nanotube-based NEMS devices-part II: role of finite kinematics, stretching and charge concentrations. J. Appl. Mech. 72, 726-731 (2005)

17. Nayfeh, A.H.: Nonlinear Interactions. Wiley Interscience, New York (2000)

18. Yakobson, B., Brabec, C., Bernholc, J.: Nanomechanics of carbon tubes: instabilities beyond linear response. Phys. Rev. Lett. 76, 2511-2514 (1996)

19. Harik, V.M.: Ranges of applicability of the continuum beam model in the mechanics of carbon nanotubes and nanorods. Solid State Commun. 120, 331-335 (2001)

20. Younis, M.I., Abdel-Rahman, E.M., Nayfeh, A.H.: A reduced-order model for electrically actuated microbeambased MEMS. J. Microelectromech. Syst. 12, 672-680 (2003)

21. Younis M. I., MEMS Linear and Nonlinear Statics and Dynamics, Springer, 2011.

22. Ruzziconi, L., Younis, M. I., and S. Lenci,

"Multistability in an electrically actuated carbon nanotube: a dynamical integrity perspective," Nonlinear Dynamics, DOI 10.1007/s11071-013-0986-5, Vol. 74, pp. 533-549, 2013. 